\title{
FIB Cross-sections of Cells on a Patch Clamp Chip
}

\author{
Jeff Fraser $^{1}$, Danish Salim ${ }^{3}$, Christophe Py ${ }^{1}$, Dolores Martinez ${ }^{1}$, Tanya Comas ${ }^{2}$, Robert Monette ${ }^{2}$ \\ ${ }^{1}$ National Research Council Institute for Microstructural Sciences, 1200 Montreal Rd, Ottawa ON \\ K1A 0R6, Canada, \\ 2 National Research Council Institute for Biological Sciences, 100 Sussex Dr \\ Ottawa, Ontario, K1A 0R6 Canada \\ 3 University of Ottawa, 75 Laurier Ave E, Ottawa ON Canada, K1N6N5
}

Patch-clamp chips developed at NRC integrate and miniaturize the traditional glass pipette on chip to allow the measurement of the electrophysiological activity of cells as in-vitro disease models with very high fidelity [1]. Paramount to the quality of the measurement is the seal between the cell and a microhole that probes it, which can be obtained if the cell is lower in the hole, forming what's called a bleb [2]. While the bleb is routinely observed in glass pipette patch-clamping, it had never been observed on a patch-clamp chip. We developed a method by which a cell cultured on a patch-clamp chip is fixed and dried in formalin, then sectioned by Focused Ion Beam [3] and finally pictured by Scanning Electron Microscopy. The striking results provide the first visual evidence of the bleb formed by a cell on a patch-clamp chip and were used to optimize the hole profile of the chip

\section{Challenges:}

- It is extremely difficult to locate the microhole from the top side of the sample due to the cells which form a confluent layer on the surface.

- Once the microhole of interest is located on the backside, how does one register the position on the top side?

\section{Solution:}

- The microhole can be located on the backside of the substrate at the bottom of the well (Figure 1).

- The position can be registered by drilling $5 \mu \mathrm{m}$ pilot holes (Figure 2) from the backside with the Focused Ion Beam (FIB) on either side of the microhole through the substrate and cell layer. The microhole can then be located for cross-sectioning by the FIB.

- From the backside deposit a Pt bar across the Bleb and microhole $5 \mu \mathrm{m}$ wide by $1 \mu \mathrm{m}$ thick. This will protect and delineate the cellular material.

- FIB a $5 \mu \mathrm{m}$ diameter hole through the substrate and cells at each end of the Pt bar.

- Reposition sample for viewing from top side.

- Locate registration holes on top side.

- Deposit Pt bar $5 \mu \mathrm{m}$ wide by $1 \mu \mathrm{m}$ thick between the registration holes.

- Cross section using FIB.

- View section in Scanning Electron Microscope.

\section{References}

[1] C.Py et al, "A multiple recording patch clamp chip with integrated subterranean microfluidic channels for cultured neuronal networks", Micro-Total Analysis Systems, San Diego CA, Oct 12-17, 2008. 
[2] J. Dunlop et al " High-throughput electrophysiology: an emerging paradigm for ion-channel screening and physiology" (2008) Nat. Rev. Drug Discov. 7(4)358

[3] L. Giannuzzi et al "Introduction to Focused Ion Beams" (2005) Springer

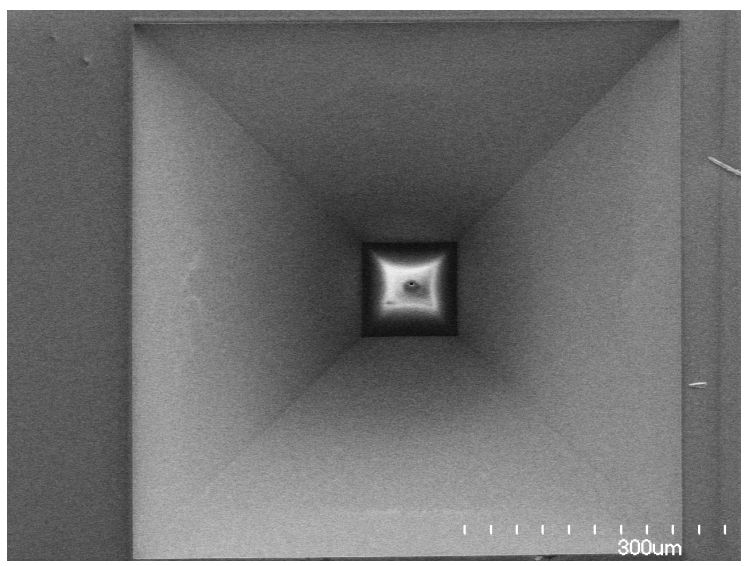

Figure 1: SEM of the backside of the chip showing the well and FIB cut.

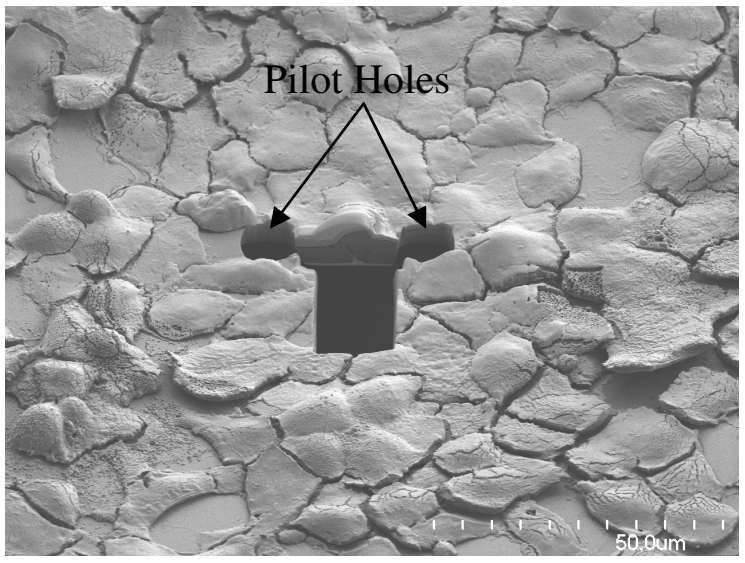

Figure 2: SEM of cell-covered patch-clamp chip following backside registration holes drilling and FIB section of the microhole-attached cell.

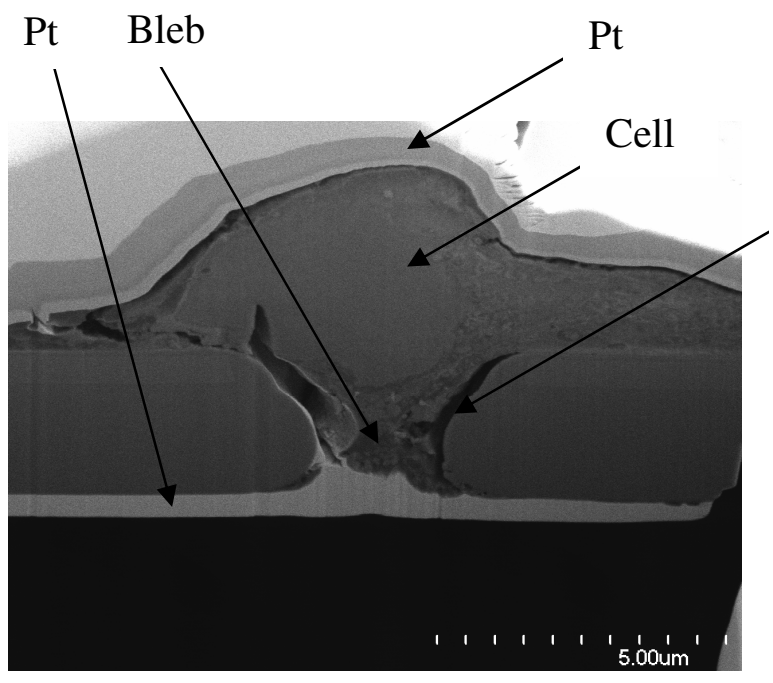

Microhole

Figure 3: SEM close-up the microhole-attached cell of interest. The cell is shown to form a bleb in the chip's microhole. 